\title{
Neuroendocrine Convertase 1
}

National Cancer Institute

\section{Source}

National Cancer Institute. Neuroendocrine Convertase 1. NCI Thesaurus. Code C127854.

Neuroendocrine convertase 1 (753 aa, $~ 84$ kDa) is encoded by the human PCSK1 gene.

This protein plays a role in the proteolytic activation of protein hormones and neuropeptides. 